\title{
Aerodynamic Character of Partial Squealer Tip Arrangements In An Axial Flow Turbine Part I : Detailed Aerodynamic Field Modifications via Three Dimensional Viscous Flow Simulations Around Baseline Tip
}

\author{
Levent Kavurmacioglu \\ Debashis Dey \\ Cengiz Camci
}

\begin{abstract}
This paper deals with the viscous flow simulations of the complex tip leakage flow in Axial Flow Turbine Research Facility (AFTRF). Special attention is paid to the 3D structure of the tip leakage flow mechanisms in a baseline tip configuration with no desensitization. Although past experimental studies provide much insight into the physical understanding of the tip region aerodynamics, there are still many areas of the flow-field in which experiments are extremely difficult to perform. Fine details of the entrance flow near the pressure side where the tip leakage jet starts to form, the leakage jet formation between the tip surface and outer casing, the re-circulatory flow zone very near the pressure side corner, the interaction area of the tip vortex with the conventional passage flow, the influence of the relative motion of the outer casing and leakage flow reversal can be visualized with excellent resolution. After the presentation of the measured inlet boundary conditions and a grid independency study, the baseline tip flow simulations are discussed in detail.
\end{abstract}

Keywords: tip leakage flow, turbine, CFD, flow visualizations.

\section{Biographical notes:}

L. Kavurmacioglu received his $\mathrm{PhD}$ in Mechanical Engineering from Istanbul Technical University in 1996. He is an Assistant Professor at the Faculty of Mechanical Engineering at Istanbul Technical University, Turkey. His current research interests include flow and heat transfer in Turbomachinery, transient flow and surge in turbomachinery systems. D. Dey received his Ph.D. in Aerospace Engineering from Penn State in 2001. His doctoral research included turbine tip leakage mitigation in high pressure stages. Currently he is a combined cycle performance test engineer at General Electric, based at Schenectady, NY.C. Camci is currently a Professor of Aerospace Engineering at the Pennsylvania State University. He obtained his doctoral degree from the von Karman Institute for Fluid Dynamics in Belgium in 1985. He directed research in the Turbomachinery Aero-heat Transfer Laboratory at Penn State since 1986. He teaches theory of turbomachinery, fluid dynamics, turbulent flow, aerospace propulsion and finite element methods in fluid dynamics and heat transfer.

1

INTRODUCTION

\subsection{Tip Clearance Flow}

The spacing required between the tips of blades and the stationary casing of an axial flow turbine is a significant source of inefficiency. The leakage flow induced by the pressure differential between the pressure side and suction side of a rotor tip usually rolls into a vortical structure. Total pressure losses at the exit of a turbine stage are directly proportional with the tip gap size. The leakage flow mixing with the rotor passage flow also reduces turbine stage efficiency.
Tip leakage related losses might account for as much as a third of the total losses in a stage. Because of the extremely small length scales involved and highly complex 3D, viscous, unsteady, turbulent flow structures, tip gap flows have always been challenging to turbomachinery researchers. Turbine tip gap leakage fluid passes through a tip gap region without experiencing a significant expansion and cooling that is typical in the core section of a turbine passage. The leakage fluid with relatively high total temperature flowing through turbine tip gaps can create important turbine durability and endurance problems. Although there are many past experimental studies aimed at understanding the tip vortex flow and heat transfer problem, there is still a need for 
reasonably accurate three-dimensional viscous flow visualizations in this region. A computational visualization approach used for the tip vortex problem is attractive because of its ability to reveal local threedimensional flow details that are extremely difficult to measure in inherently small tip gap region.

\subsection{Flow Visualizations via Computations}

A numerical simulation of compressible flow near a turbine tip gap region in a linear cascade arrangement was presented by (Liu and Bozzola 1993). A significant reduction

of gap exit leakage mass flow from a moving outer casing was presented in comparison to a stationary outer wall. (Basson and Lakshminarayana, 1993) implemented an embedded grid generation method into a three

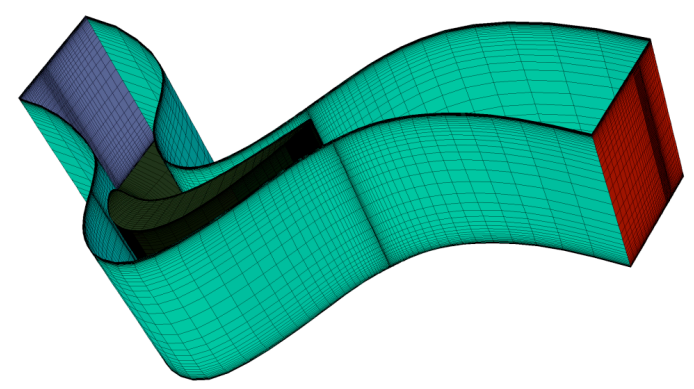

Figure $13 D$ computational grid for the AFTRF turbine rotor flow simulations

dimensional pressure-based semi-implicit scheme for the prediction of tip clearance flows. (Sell et al, 2000) presented computational tip aerodynamics results from a linear turbine cascade with an exit Mach number of 0.5 . They reported that the computational simulations agree well with their measurements. The effects of tip clearance height and casing relative motion in axial turbines were investigated by (Tallman and Lakshminarayana, 2001a; 2001b) and (Morphis and Bindon, 1988). They reported that the structure of aerodynamic losses in the turbine passage change dramatically when the outer casing motion was incorporated into the analysis. (Ameri et al., 1998) computationally investigated the effect of tip recess on tip heat transfer and efficiency. They found that the numerical prediction of the effect of the casing recess on blade and tip heat transfer and efficiency was reliable.

(Bunker, Bailey and Ameri, 2000) obtained tip heat transfer and pressure measurements in a three bladed linear cascade simulating the first stage geometry from a large power generating turbine with flat and smooth tip surfaces. They noticed a central "sweet spot" of low heat transfer extending into the mid chord region and toward the suction side. Measured surface heat transfer coefficients increased $10-20 \%$ when free stream turbulence intensity level was increased from 5 to $9 \%$. When the sharp tip edge was rounded, the tip heat transfer increased by about $10 \%$, presumably due to higher allowed tip leakage flow. (Bunker and Ameri, 2000) also published the results of a study dealing with the numerical prediction of the tip gap heat transfer problem defined in (Bunker, Bailey and Ameri, 2000). The casing upstream of the blade was recessed. The numerical results with a radiused-edge blade agreed better with the experimental data. They attributed this feature to the absence of a separation bubble usually forming in the gap region. (Lin et al., 2000) studied the effects of gap leakage on fluid flow in a contoured turbine nozzle guide vane in a computational study. A numerical analysis of tip vortex flow in an annular turbine cascade configuration was performed by (Han et al., 2001)

\subsection{Current objectives}

The present investigation deals with the aerodynamic visualization of tip leakage flow existing in the baseline tip configuration of the AFTRF. The computational visualizations are obtained from three-dimensional

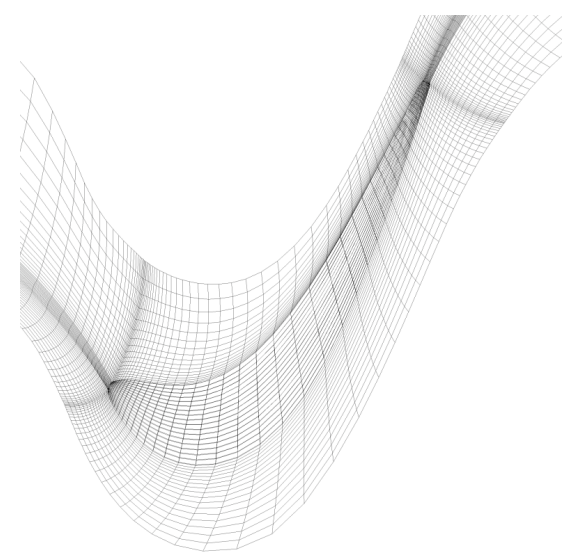

Figure 2 Grid structure near the baseline tip configuration

turbulent flow simulations using a general purpose Reynolds Averaged Navier-Stokes solver. The computational study makes extensive use of carefully measured inlet boundary conditions to the rotor. The details of the turbine facility and the recent stage exit measurements for a baseline tip configuration are presented in (Lakshminarayana, Camci, Halliwell \& Zaccaria, 1992), (Camci, 2004), (Dey and Camci, 2001) and (Camci, Dey and Kavurmacioglu, 2003), respectively. The numerically visualized tip gap flow field from the RANS simulations could be effective in explaining local three-dimensional flow details in turbine passages where aerodynamic measurements are extremely difficult to perform. Numerically generated "surface oil flow visualizations" on the tip surface and predicted "vortical flow details" on user-defined planes could be effectively used to study local tip flow physics. The "vortical flow details" in user-defined planes are numerical equivalents of "laser sheet visualizations" frequently used in wind 
tunnel studies. The study clearly shows that the turbine tip gap region includes many different leakage flow regimes. The leakage flow characteristics are usually controlled by the effective tip clearance, tip geometry, local

aerodynamic loading conditions and the rotational speed of the rotor.

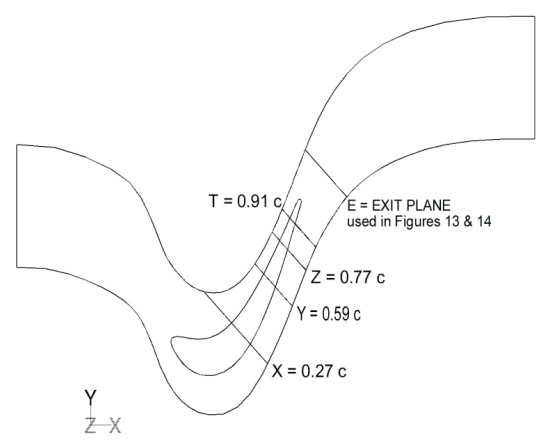

Figure 3 Tip leakage visualization planes (cross-stream direction $\&$ blade height)

\section{NUMERICAL ANALYSIS}

\subsection{Governing Equations}

The numerical simulations of the steady-state 3D flow field inside the turbine rotor passage with tip clearance are obtained by solving the three-dimensional and incompressible Reynolds Averaged Navier-Stokes equations. Figure 1 shows the 3D linear cascade equivalent of the annular turbine blade passage in AFTRF. The continuity equation and momentum equations in tensor notation are:

$U_{i, i}=0$

and $\rho U_{j} \frac{\partial U_{i}}{\partial x_{j}}=-\frac{\partial p}{\partial x_{i}}+\mu \frac{\partial^{2} U_{i}}{\partial x_{j} \partial x_{j}}+\frac{\partial R_{i j}}{\partial x_{j}}$

where $R_{i j}=-\rho \overline{u_{i} u_{j}}$ are the Reynolds Stresses. The Reynolds stresses are modeled by using the Boussinesq hypothesis.

$R_{i j}=-\rho \overline{u_{i} u_{j}}=-\rho \frac{2}{3} k \delta_{i j}+\mu_{t}\left(\frac{\partial U_{i}}{\partial x_{j}}+\frac{\partial U_{j}}{\partial x_{i}}\right)$

For two-equation models, the turbulent viscosity is related to turbulent kinetic energy $k=\overline{u_{i} u_{i}} / 2$ and the dissipation rate $\varepsilon$ as $\mu_{t}=\rho C_{\mu} k^{2} / \varepsilon$. The turbulent kinetic energy equation is as follows,

$\rho U_{i} \frac{\partial k}{\partial x_{i}}=\mu_{t}\left(\frac{\partial U_{j}}{\partial x_{i}}+\frac{\partial U_{i}}{\partial x_{j}}\right) \frac{\partial U_{j}}{\partial x_{i}}+\frac{\partial}{\partial x_{i}}\left\{\left(\mu+\mu_{t} / \sigma_{k}\right) \frac{\partial k}{\partial x_{i}}\right\}-\rho \varepsilon$

$$
\begin{aligned}
& \rho U_{i} \frac{\partial \varepsilon}{\partial x_{i}}=C_{1 \varepsilon} \frac{\varepsilon}{k} \mu_{t}\left(\frac{\partial U_{j}}{\partial x_{i}}+\frac{\partial U_{i}}{\partial x_{j}}\right) \frac{\partial U_{j}}{\partial x_{i}}-C_{2 \varepsilon} \frac{\varepsilon^{2}}{k} \\
& +\frac{\partial}{\partial x_{i}}\left\{\left(\mu+\mu_{t} / \sigma_{\varepsilon}\right) \frac{\partial \varepsilon}{\partial x_{i}}\right\}
\end{aligned}
$$

where

$\sigma_{k,}=1, \sigma_{\varepsilon}=1.3, C_{1 \varepsilon}=1.44, C_{2 \varepsilon}=1.92$

are empirical constants.

\subsection{Near Wall Modeling}

The most widely utilized wall functions based on (Launder and Spalding, 1974) are used for the near wall treatment. The law-of-the-wall for mean velocity is,

$\frac{U_{p} C_{\mu}^{1 / 4} k_{p}^{1 / 2}}{\tau_{w} / \rho}=\frac{1}{\kappa} \operatorname{Ln}\left(E y_{p}^{*}\right)$

where $y_{p}^{*}=\rho C_{\mu}^{1 / 4} k_{p}^{1 / 2} y_{p} / \mu$ and $\left(y_{p}, k_{p}\right)$ are the normal distance from wall to cell center and turbulent kinetic energy at wall-adjacent cell, respectively. The lawof-the-wall for mean velocity is based on wall unit $\mathrm{y}^{*}$ instead of $y^{+}=\rho u_{\tau} y / \mu$ since these are approximately equal to each other in equilibrium turbulent boundary layers.

\subsection{Method of Solution}

The numerical solutions of equations 1 through 5 with proper boundary conditions were carried out by using a finite volume technique based general purpose viscous flow solver. QUICK discretization scheme was preferred for improved accuracy. SIMPLEC algorithm was selected for the pressure-velocity coupling in order to improve convergence for such a complicated turbulent flow. A multi-grid scheme was used to solve the discretized equations in order to accelerate the convergence of the solver.

\subsection{Boundary Conditions:}

All of the computations are performed for $\mathrm{Re}=291,000$ that are based on axial chord length at the tip diameter and mass averaged relative inlet velocity to the rotor passage in the turbine facility. The specific relative velocities from the established velocity triangles of the AFTRF rotor are used, (Camci, 2004). Figure 4 shows the measured and design values of the axial, tangential and radial velocity components measured just upstream of the turbine rotor in AFTRF. The velocity magnitude and boundary layer profiles at the "inlet section" of the three-dimensional computational domain shown in Figure 1 are specified from careful measurement in AFTRF. The values of $k$ and $\varepsilon$ that are imposed at the inlet boundary are based on measured turbulence intensity $T u_{\infty}=\sqrt{2 k / 3} / U_{\infty}$ and

The dissipation rate equation is, 
length scale $L=C_{\mu} k_{p}^{3 / 2} / \varepsilon$. The measured values of $T u_{\infty}$ and $L$ from the AFTRF are used.

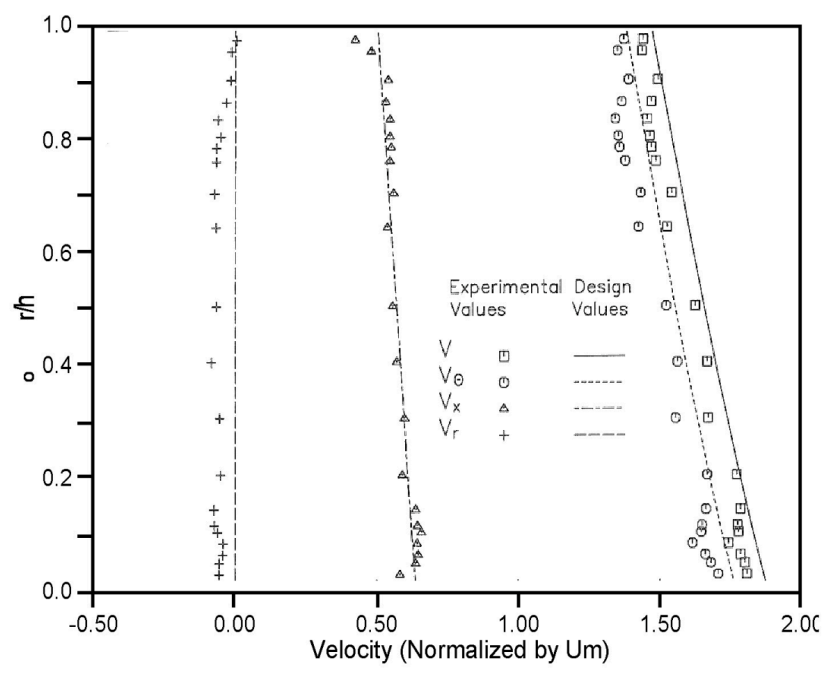

Figure 4 Measured and design values of turbine rotor inlet flow conditions

Velocities, turbulent kinetic energy and the dissipation rate are all set to zero on solid boundaries except on the outer casing. The tangential velocity of the outer casing that is used for the simulation of casing relative motion is set to a constant. The relative velocity of the outer casing in tangential direction is computed from the turbine rotational speed $(\mathrm{N}=1320 \mathrm{rpm})$ at the outer casing diameter $(\mathrm{D}=0.914 \mathrm{~m})$. The outlet boundary is set at three chord length distance from the trailing edge to ensure that there is no influence of the outlet boundary on the flow structure inside the domain. The streamwise gradients of all variables are set to zero for outlet boundary conditions. The steady mean flow through an axial turbomachinery blade row can be modeled as periodic in the circumferential direction. Figure 1 shows the periodic boundaries of the domain in circumferential direction in three dimensional space.

\subsection{Grid Structure:}

The grid structure shown in Figures 1 and 2 was generated using a general purpose grid-generating program known as GAMBIT. Two different block structured grids that have $130 \times 65 \times 88$ points (fine mesh) and $105 \times 51 \times 74$ points(medium mesh) in axial, pitchwise and spanwise directions were generated after initial experiments with a relatively coarser grid. The grid was clustered near the leading edge, trailing edgeand near the tip region. The $\mathrm{y}^{+}$ value along the spanwise direction is $30<\mathrm{y}^{+}<60$ near the hub, blade tip surface and casing. The flow field results obtained from the medium mesh and fine mesh were almost identical at the blade mid span and tip location. Figure 2 shows a typical grid structure used for the tip region of the AFTRF baseline configuration. $75 \times 65 \times 26$ and $50 \times 51 \times 26$ grid points were used for the fine mesh and medium mesh inside the tip region respectively. The numerical results were displayed on planes that are almost normal to the turbine blade tip platform. The plane definitions in the computational domain are included in Figure 3.

\subsection{Static Pressure Field on the Baseline Tip Platform:}

Figure 5 shows the static pressure distribution on the baseline tip platforms BS100 and BS33. The nondimensional tip gap for BS100 is $\mathrm{t} / \mathrm{h}=1.03 \%$ where $\mathrm{h}=123$ $\mathrm{mm}$. The tip gap for BS33 is one third of that of BS100. BS 100 forms the largest tip gap studied in this investigation (approximately the nominal tip clearance in AFTRF). BS33 is the minimum tip gap used for comparative purposes. The $\mathrm{C}_{\mathrm{p}}$ distribution for $\mathrm{BS} 100$ shows a (red-yellow) "high pressure zone" in the first $20 \%$ chord distance measured from the leading edge. Between $20 \%$ and $35 \%$ chord distance, a green intermediate pressure zone appears just before the portion of the blade where there is a significant amount of leakage flow from the pressure side to the suction side of the blade. The dark blue $\mathrm{C}_{\mathrm{p}}$ zone marks the "dominant leakage zone" in which most of the fluid leaking from the pressure side to suction side is contained (blue zone). The last $20 \%$ chord of the blade is dominated by a "relatively low leakage zone" as marked by the green zone in BS100. The insets in Figure 5 show the $\mathrm{C}_{\mathrm{p}}$ line distribution on the suction side and pressure side of the tip platform. The free stream velocities on both the pressure side and suction side of the blade reach to almost identical levels resulting in very similar chordwise $\mathrm{C}_{\mathrm{p}}$ distributions on both sides of the blade in the last $20 \%$ chord.

BS33 presented in Figure 5 shows the spread of the redyellow "high pressure" zone up to the first $40 \%$ chordwise distance from the leading edge. Since the tip gap is one third of that of BS100, the low momentum fluid contained in this area is not expected to generate significant leakage flow into the suction side. Relatively low velocities are expected in this zone. The static pressure level indicated by the green color after the first $40 \%$ chord in the BS33 case is about 1.8 times higher than the static pressure in the BS100 case at the same location.

A reduced leakage flow consistent with this elevated pressure region (green) is expected in BS33. The narrow baseline gap also shows a gradual increase in $\mathrm{C}_{\mathrm{p}}$ in the last $20 \%$ chord distance near the trailing edge. The insets in Figure 5 indicate that the pressure differential between the suction side and pressure side is minimal in this zone.

\subsection{Velocity Field in Planes Parallel to Baseline Tip Surface:}

Figure 6 shows the velocity vectors in two selected planes parallel to the blade tip platform. 

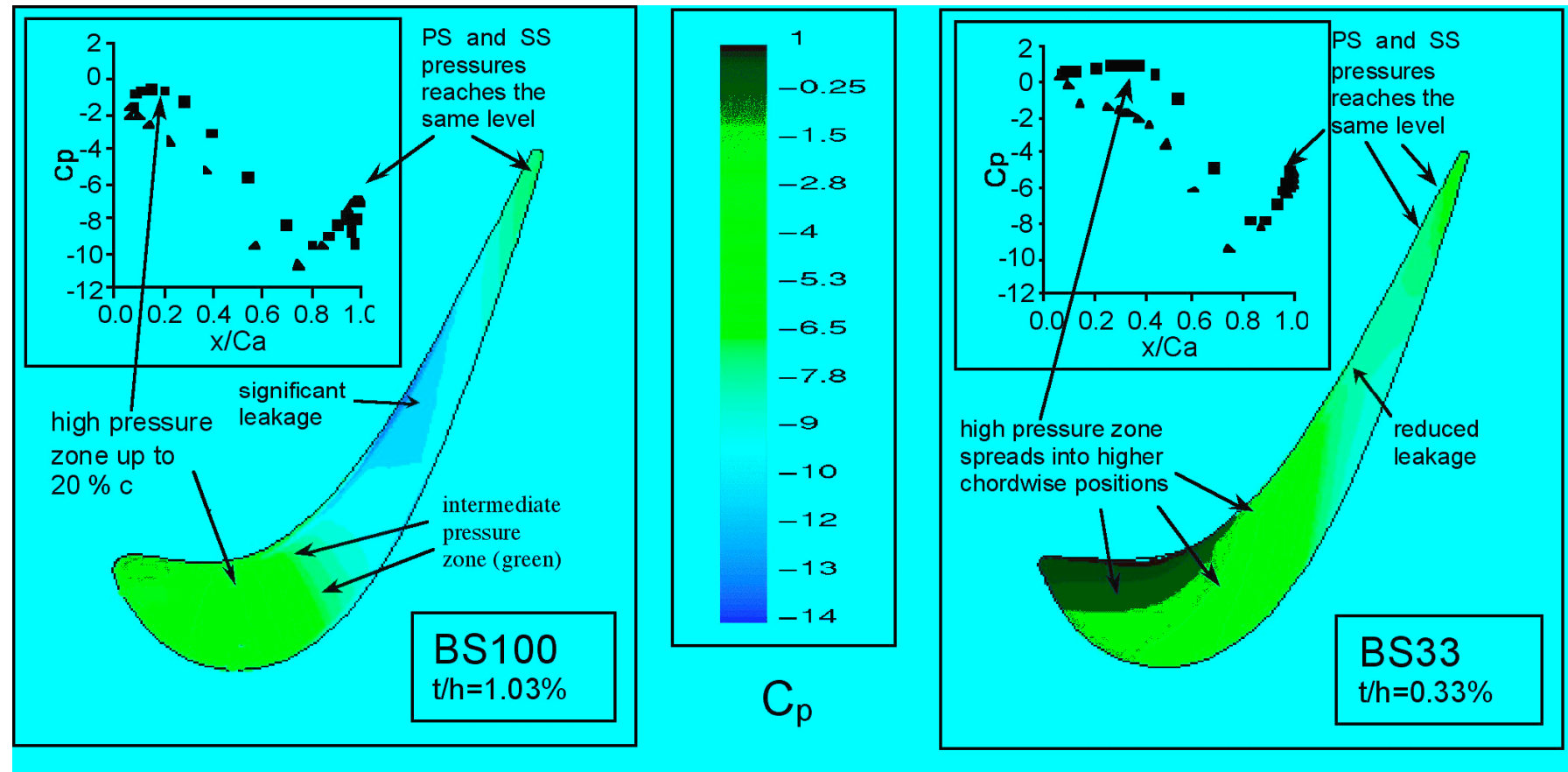

Figure 5 Static pressure distributions on the tip surface on the blade (BASELINE TIP)

The "lower plane" at $\mathrm{z} / \mathrm{t}=1 / 3$, contains the velocity vectors near the tip platform mainly dominated by the pressure driven flow. The "higher plane" at $\mathrm{z} / \mathrm{t}=5 / 6$ shows the velocity vectors in a plane very close to the outer casing that is inducing a significant shear effect on the leakage flow in the gap. For the baseline case BS100 the general trends in terms of the velocity magnitude and direction are similar in the lower plane and higher plane. Near the blade tip platform, most of the flow originating near the pressure side corner tends to pass to the suction side. The strongest leakage velocities are observed as orange-red vectors near the pressure side corner, after 40 $\%$ chord distance from the leading edge. The fluid particles from the passage accelerate into the tip gap. The trailing edge leakage flow vector magnitudes are not as large as the ones in the mid-chord region. The velocity direction is in blade camber-line direction and this flow does not actively participate in the formation of the loss generating vortical system termed as tip vortex. When one moves to the higher plane near the outer casing, a similar flow picture is evident. The outer casing influence in general works against the leakage flow in the tip gap. The upper right figure in Figure 6 shows the slight turning of the velocity vectors towards the pressure side because of casing induced shear. This turning is less severe in locations where the pressure difference between the pressure side and the suction side is strong. The turning of the vectors because of the outer casing shear is more significant in regions where the driving pressure difference is minimal between the PS and SS. A more apparent turning of the flow in the upper plane is apparent near the leading edge zone and in the last $20 \%$ chord of the blade.
The lower left and right pictures in Figure 6 show the velocity vectors when the tip gap is small $(\mathrm{t} / \mathrm{h}=0.33 \%)$. The most apparent observation is the strong influence of the outer casing motion that is felt similarly in both lower visualization plane and upper plane because of the tight clearance. When compared to BS100, the velocity vectors in the planes of the narrow gap termed BS33, there is at least $20^{\circ}$ to $30^{\circ}$ directional change towards the pressure side (a counter clockwise turn). When the leakage flow forms in the narrow gap, the outer casing has a tremendous ability to pull the fluid layers in a direction opposite to the typical leakage direction. It is interesting to note that the high pressure zone indicated by a red-yellow color in Figure 5 contains an extremely low momentum fluid in the first $20 \%$ of the chord length. The leakage flow in this region is from the suction side to pressure side at a very low velocity. The suction side of the blade does not contribute to a strong tip vortex formation. The boundary IJ for the lower plane in BS33 marks the location where the measurable leakage to the suction side is initiated. Some of the fluid entering from the suction side may travel inside the tip gap for a while before it joins the weak tip vortex formation at before point $\mathrm{J}$.

Near the trailing edge, the leakage direction is clearly reversed. Because of the extremely narrow tip gap, in the trailing edge region, the tip gap flow is severely turned back into the pressure side of the channel via turbulent shear action. A comparison of BS100 and BS 33 shows a weakening of the tip vortex by just designing the tip gap in an extremely tight manner 

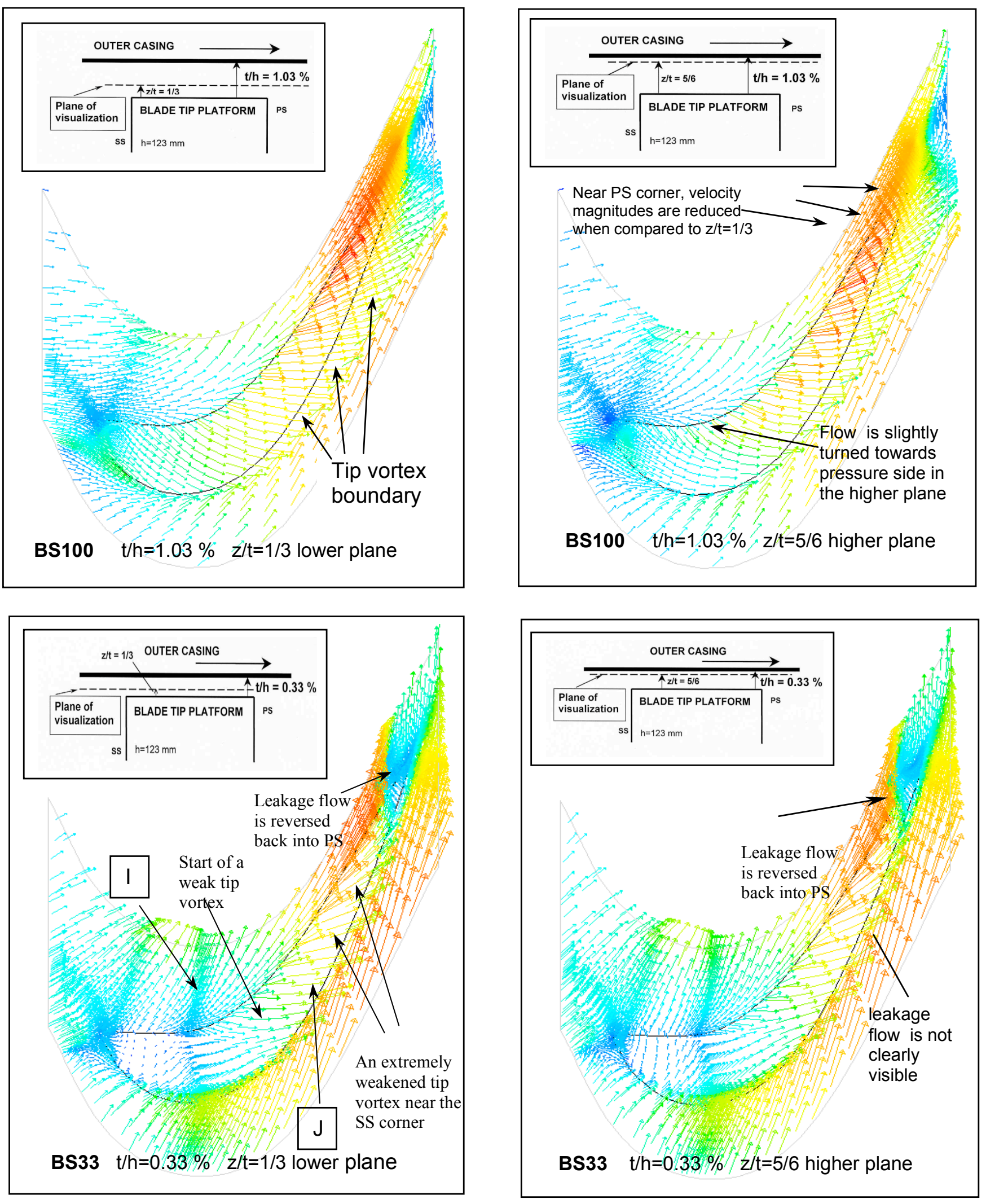

Figure 6 Velocity vectors at various planes in the tip gap (BASELINE TIP) 


\subsection{Re-circulatory Tip Flow Patterns in Cross-stream Planes:}

Figure 7 shows numerically generated flow visualization patterns inside planes (X,Y,Z and $\mathrm{T}$ ) defined by the cross stream direction and radial direction. The visualizations were generated by drawing pathlines using the velocity components inside the visualization planes. This type of numerical visualization is equivalent to smoke flow visualizations performed in laser sheets of visible light in wind tunnels. A clear leakage flow from the pressure side to suction side is apparent for the baseline gap BS100 at plane $X(0.27 \% \mathrm{c})$. The outer casing effect in this zone is not detectable. Most of the fluid leaking in this zone approaches the pressure side corner in a radially outward direction. An extremely small separation bubble is expected near the pressure side corner. In plane Y $(0.59 \%$ c), leakage flow character is the same as $\mathrm{X}$, however, the tip vortex on the suction side starts growing at a faster rate. Plane $Z(0.77 \% \mathrm{c})$ is interesting because of the start of a major directional change of the velocity vectors trying to turn into the pressure side. The leakage flow suddenly looses its driving pressure differential in the last 20-25\% of the trailing edge as shown in the $\mathrm{Cp}$ distributions of Figure 5. The turbulent shear action in this region starts pulling some of the tip gap fluid back to the pressure side in region $M$. A counter clockwise vorticity is induced near the pressure side of the gap. In addition to some weak leakage to the suction side, a highly circulatory bubble (M) forms in the gap. In plane $\mathrm{T}$ that is located in the last $10 \%$ of the chord, this flow reversal process is complete. A full reversal of the leakage flow from the suction side to pressure side is observed. Some of the fluid trapped inside the tip vortex structure near the suction side $(\mathrm{N})$ can go back to the tip gap region, eventually crossing to the pressure side of the passage. The right hand side column in Figure 7 shows the pathlines inside the visualization planes for the small tip gap termed as $\mathrm{BS} 33, \mathrm{t} / \mathrm{h}=0.33 \%$. In plane $\mathrm{X}$, although the gap is extremely tight, a weak leakage originates from the pressure side to suction side. The tip vortex grows quite slowly compared to BS100 as one moves to the trailing edge. The cross section of the leakage vortex near the suction side is much smaller than the case for BS100. The leakage flow is completely reversed in plane $\mathrm{Z}$ showing the strong viscous/turbulent shear effect imposed by the outer casing in a zone where the pressure differential between the pressure side and suction side starts to diminish. If the visualization plane $\mathrm{Z}$ for BS100 is examined, it is noticed that only a partial reversal to pressure side is apparent. The viscous/turbulent shearing effect of the outer casing starts dominating at a much faster rate when the clearance is tight.

The computed leakage mass flow rate for BS100 passing through the suction side boundary of the tip gap is about $0.0081 \mathrm{~kg} / \mathrm{s}$. When the clearance is reduced three times, the leakage mass flow rate is reduced by factor of 11 . The computed leakage $\mathrm{Re}_{\text {tip }}$ number based on tip gap height for BS100 is $\mathrm{Re}_{\text {tip }}=2824$ and $\mathrm{Re}_{\text {tip }}=262$ for BS33. The velocity in computed leakage $\mathrm{Re}_{\text {tip }}$ number was calculated from the mass flow rate computation in the suction side leakage area.

There is a remnant of the original counter clockwise rotating "weak" tip vortex (formed previously), near the suction side corner in plane $Z$. In plane $T$, the tip gap flow is completely reversed from the suction side to pressure side. At this location very near the trailing edge, the original "weak" tip vortex is not visible anymore. A slight curvature of the pathlines near the suction side corner is all that is visible. Figure 7 clearly demonstrates the desensitization of a large tip vortex area that may contain a tremendous momentum deficit and energy loss in the turbine passage by just reducing the tip gap space.

\subsection{Leakage Flow Patterns in Planes Parallel to Tip Surface:}

Figure 8 shows the pathlines for tip gap flows in two different planes $(\mathrm{z} / \mathrm{t}=1 / 3$ and $5 / 6)$ for the baseline cases of BS100 and BS33. The fluid particles entering the tip gap space on the suction side near the leading edge follow a path either very close to the blade boundary near the suction side $(\mathrm{P})$ or in the middle section $(\mathrm{R})$ of the leading edge before the location $\mathrm{X}=0.27$.c. The leakage paths for the particles originating from the pressure side corner are well defined. The particles tend to form a clear boundary for the tip vortex in the passage (S). The pathlines are only slightly different in the higher plane at $\mathrm{z} / \mathrm{t}=5 / 6$ (upper-left frame) because of the outer casing pulling effect via viscous/turbulent shear in a direction opposite to a typical leakage direction. The pathlines in the first $1 / 3$ of the blade slightly turn towards the pressure side in the higher plane.

When the tip gap is small, a leading edge and trailing edge modification of leakage flows occur due to the outer case motion in regions where driving pressure differentials are small. In both the lower and higher visualization plane in Figure 8, the fluid from the suction side crosses the gap in a direction towards the pressure side near the leading edge (T) and trailing edge (U). Some of the fluid particles near the suction side corner enter the tip gap space only to leave it after turning back to suction side. They usually mix with the "weak" tip vortex forming near the suction side corner. Figure 8 shows that the outer boundaries of the tip leakage vortex for BS 33 is much smaller than that of BS100 that has three times the tip gap height. The weak tip vortex for this case suddenly turns into pressure side of the channel (U1). The leakage flow reversal shown in the vertical visualization planes $\mathrm{Z}$ and $\mathrm{T}$ in Figure 7 may form a "secondary reversed leakage vortex" (U2) discharging into the pressure side of the blade near the trailing edge. Because of the extremely narrow tip gap in $\mathrm{BS} 33$, in the trailing edge region where there is almost no potential for leakage; the tip gap flow is turned back into the pressure side of the channel via viscous/turbulent shear action. A comparison of BS100 and BS 33 shows the effective weakening of the tip vortex by just designing the 


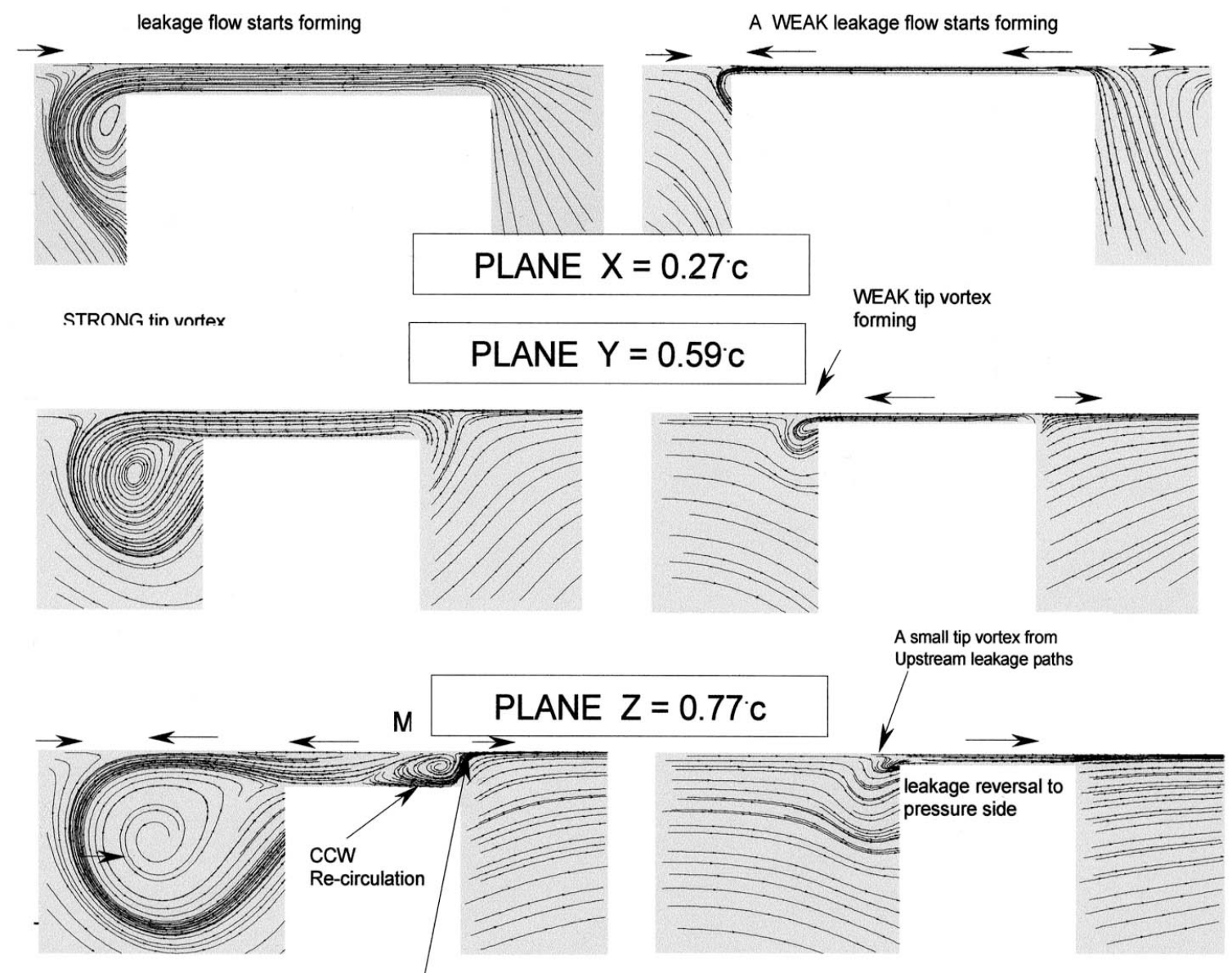

Viscous/turbulent shear from the outer casing motion starts pulling the tip gap fluid back to PS

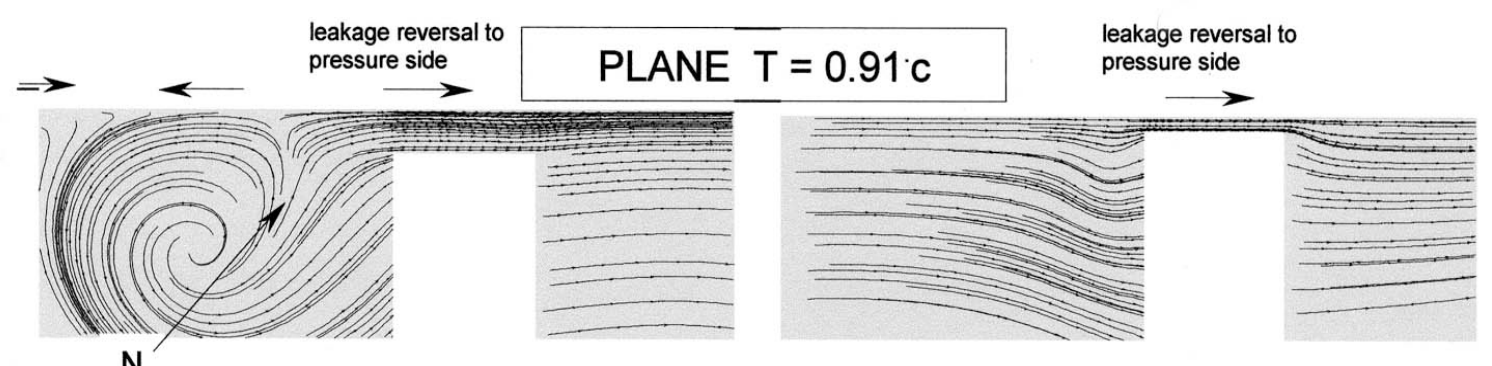

Some of the fluid trapped inside the tip vortex may go back to the tip gap (eventually to cross to PS

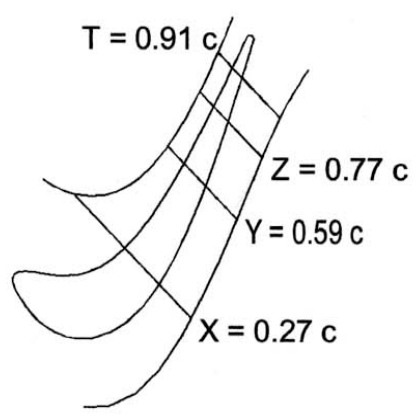

Figure 7 Leakage Flow Patterns in the tip gap space inside cross stream planes 

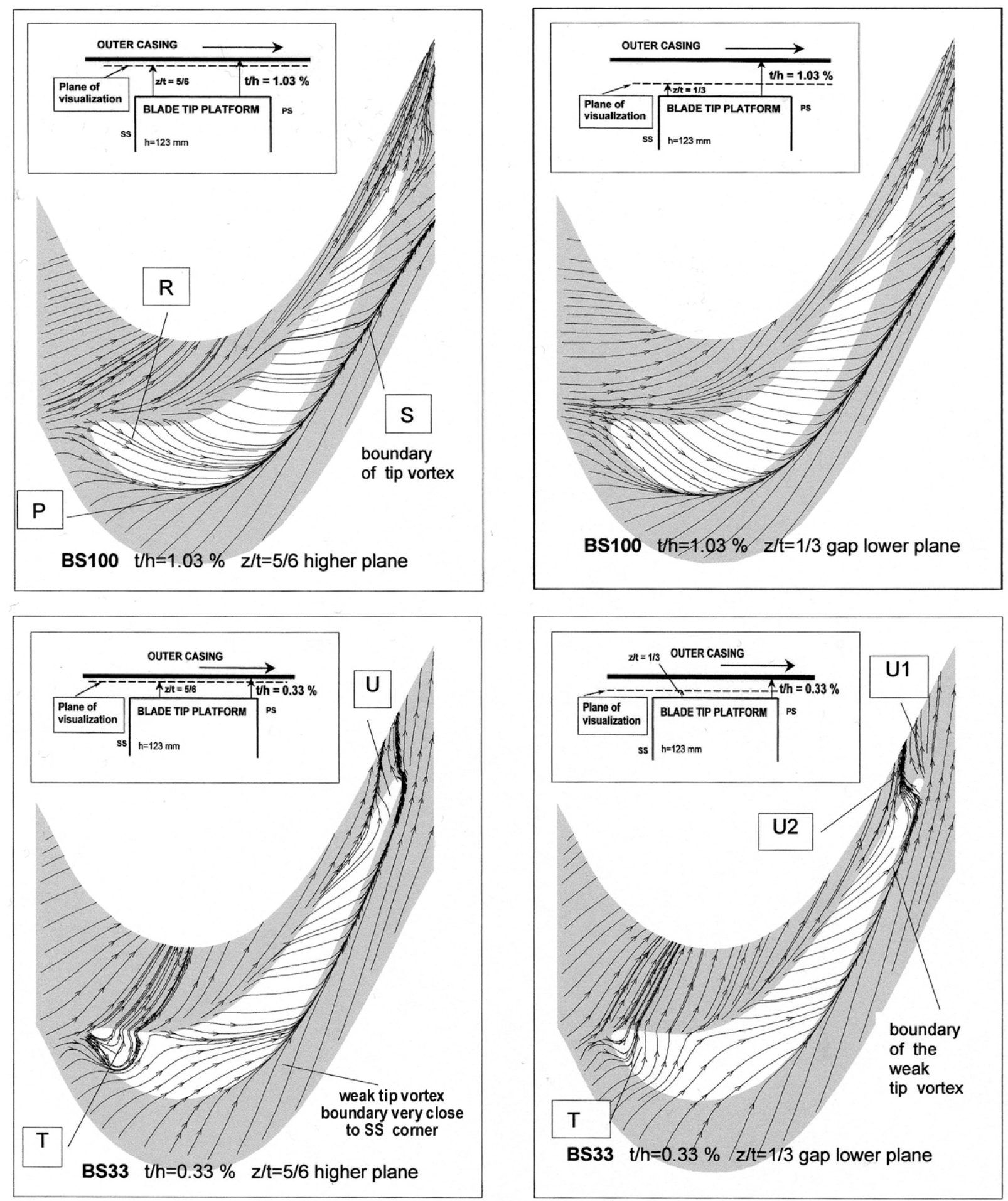

Figure 8, Leakage flow patterns in planes parallel to the tip surface, (BASELINE TIP, full cover) 
tip gap in an extremely tight manner. Although not practical for actual turbine operation, BS33 forms a baseline case for comparative purposes in this study. It is expected that when the tight clearance of BS33 is used "only" in a very narrow region on top of a partial squealer rim, similar flow physics should contribute to the success of the de-sensitization process. Partial squealer tip visualizations are provided in Part-2 of this paper.

\section{CONCLUSIONS}

3D viscous flow simulations of the complex tip leakage flow-field existing in the Axial Flow Turbine Facility (AFTRF) are presented for the baseline tip configuration. Although past experimental studies provide much insight into the physical understanding of the tip region aerodynamics, there are still many areas of this flow-field in which experiments are extremely difficult to perform.

The general attributes of the physical aspects of the leakage flows on the baseline tip configurations are successfully simulated in a numerical visualization effort.

Fine details of the entrance flow near the pressure side corner where the tip leakage jet starts to form, the leakage jet formation between the pressure side and the suction side, the re-circulatory flow zone near the pressure side corner in the tip gap zone, the interaction area of the tip vortex with the conventional passage vortex system, the influence of the relative motion of the outer casing and leakage flow reversal can all be visualized in detail by using computational tools solving the three-dimensional Reynolds Averaged Navier-Stokes Equations.

The static pressure distributions obtained on the tip platform surface for two different clearance values reveal many important flow features such as low momentum/high static pressure zone near the leading edge, a dominant leakage area near blade mid-chord location and a minimum pressure difference zone between the PS and SS near the trailing edge wedge area.

A strong static pressure modification is apparent when the clearance is reduced to $\mathrm{t} / \mathrm{h}=0.33 \%(\mathrm{BS} 33)$ from the baseline case of $\mathrm{t} / \mathrm{h}=1.03 \%$ (BS100). The high-pressure zone near the leading edge spread into the mid-chord region of the blade when the clearance is reduced. The velocity vectors in this zone have small magnitude compared to the dominant leakage zone. This highpressure zone coincides with the "central sweet spot" observed in cascade heat transfer measurements on similar blades by a number of researchers. Leakage flow paths in this region can be from the pressure side to suction side area as observed from the leakage flow patterns.

Velocity field visualized in planes parallel to the tip platform provides insight in terms of the direction and the magnitude of the leakage flow patterns in the tip gap zone. The viscous/turbulent shearing effect of the outer casing is clearly visible in velocity vector maps especially in the higher plane located very close to the outer casing. When the clearance is tight (BS33), the outer casing viscous/turbulent shear effect is felt even in the lower plane located very near the tip platform of the blade.

An effective visualization of the tip vortex structure for the baseline clearance (BS100) and tight clearance (BS33) is presented in vertical planes (X,Y,Z and $\mathrm{T})$. Vortical flow details in these vertical planes show that a large tip vortex structure rolling near the suction side corner may occupy a large area with significant momentum deficit and energy loss for (BS100). When the clearance is reduced to one third of that of (BS100), an effectively weakened tip vortex structure is visible.

The leakage flow direction, amount and momentum is controlled by the delicate balance between the pressure forces, the shear forces imposed by the outer casing and inertial forces resulting from convective accelerations. It is likely that the shear influence of the outer casing is dominant in blade zones where the driving pressure differentials are minimized by the tip loading conditions.

The numerical visualizations indicate that the small zone near the leading edge of the blade and the trailing edge wedge zone may have flow leakage from the suction side to pressure side. Leakage flow reversal in the last $20 \%$ chord of the blade is common. The reversal of the leakage flow occurs in the areas where the driving pressure differential along a leakage flow path between the pressure side and suction side is minimized. The pressure differential is minimal in most turbine blade trailing edge zones because of the highly accelerated passage flows on both sides of the blade are brought into similar Mach number values by design.

The viscous/turbulent shearing effect of the outer casing starts dominating at a much faster rate (earlier chordwise positions) when the clearance is tight. Strong driving pressure differential in fluid layers near the tip platform and a strong shear force imposed by the outer casing near the outer wall may create sizeable re-circulatory flow patterns in the gap especially in the second half of the blade.

The re-circulatory flow zones numerically visualized in vertical planes form as angular deviations of leakage flow from the mean camber line direction. Flow velocities are such that a typical leakage is sustained from the pressure side to suction side after the mid-chord location. However, in the last $20 \%$ chord of the blade, leakage flow reversal occurs. The leakage flow tends to turn back to the pressure side. Although clearly visible even for the baseline clearance (BS100) case, the reversal of the leakage flow is much stronger for the tight clearance case (BS33).

When tip leakage reversal occurs, some of the fluid trapped inside the conventional tip vortex (located near the suction side corner) may go back into the tip gap zone. This fluid may eventually find its way to the pressure side. Heat transfer implications of this feature need to be 
studied since the fluid trapped inside the tip vortex is likely to have higher total temperature than the core flow.

\section{NOMENCLATURE}

c Rotor axial chord length at tip $=0.129 \mathrm{~m}$

Always an axial chord is assumed troughout the paper

BS100 Baseline tip configuration with no tip treatment, full cover, $\mathrm{t} / \mathrm{h}=1.03 \%$

BS33 Baseline tip configuration with no tip treatment, full cover, $\mathrm{t} / \mathrm{h}=0.33 \%$

$\mathrm{C}_{\mathrm{p}} \quad$ Static pressure coefficient

$C_{p}=\left(p-p_{\text {ref }}\right) / 0.5 \rho W_{\text {inlet }}^{2}$

$\varepsilon \quad$ Turbulent dissipation rate

h $\quad$ Rotor blade height $=0.123 \mathrm{~m}$

k Turbulent kinetic energy

$\kappa \quad$ Von Karman constant

$\mathrm{p}_{\mathrm{o}} \quad$ Total pressure

$\mathrm{p}_{\mathrm{atm}} \quad$ Ambient pressure (also pref)

$\mathrm{p}_{\text {in }} \quad$ Inlet total pressure

$\mathrm{p} \quad$ Static pressure

PS, SS Pressure side, suction side

Re Reynolds number

$\mathrm{Re}_{\text {tip }} \quad$ Tip leakage flow Reynolds number

$\mathrm{Re}_{\text {tip }}=\rho V_{\text {tip }} t / \mu$

$\rho \quad$ Density

$\mathrm{r} / \mathrm{h} \quad$ Non-dimensional radial position measured from hub surface (also $\mathrm{y} / \mathrm{h}$ in contour plots)

t Rotor tip clearance height

TE Trailing edge

$\mathrm{U}_{\mathrm{i}} \quad$ Mean velocity components

$\mathrm{U}_{\mathrm{m}} \quad$ Mean wheel speed at rotor mid-span

V Absolute velocity

W Relative velocity with respect to turbine rotor

$\mathrm{x}, \mathrm{y}, \mathrm{z}$ Cartesian coordinate system for the numerical analysis (axial, tangential, radial direction)

$\mathrm{X}, \mathrm{Y}, \mathrm{Z}, \mathrm{T}$ Flow visualization planes used for numerical laser sheet visualizations (see Figure 7)

$\mathrm{z} / \mathrm{t} \quad$ distance between the plane of visualization and blade tip platform (see Figure 6)

\section{REFERENCES}

Ameri, A.A., Steinthorsson, E., Rigby, L.D., 1998, "Effects of Tip Clearance and Casing Recess on Heat Transfer and Stage Efficiency in Axial Turbines," Nasa Contract Report NASA/CR-1998-208514.

Basson and Lakshminarayana, 1993, "Numerical Simulation of Tip Clearance Effects in Turbomachinery," ASME Journal of Turbomachinery, Vol.109, pp.545-549.

Bunker, R.S., Ameri, A.A., 2000,'Heat Transfer and Flow on the First Stage Blade Tip of a Power Generation Gas Turbine: Part 2-Simulation Results," ASME Journal of Turbomachinery, Vol.122, pp.272-277.

Bunker, R.S., Bailey, J.C., Ameri, A.A., 2000,'Heat Transfer and Flow on the First Stage Blade Tip of a Power Generation Gas Turbine: Part 1-Experimental Results," ASME Journal of Turbomachinery, Vol.122, pp.263-271.

Camci, C., 2004, "A Turbine Research Facility to Study Tip Desensitization Including Cooling Flows," VKI Lecture Series on " Turbine Blade Tip Design and Tip Clearance Treatment" 19-23 January 2004, pp. 1-26.

Camci, C., Dey, D., Kavurmacioglu, L., 2003, “ Tip Leakage Flows near Partial Squealer Rims in an Axial Flow Turbine Stage,", ASME GT2003-38979, paper presented at the ASME IGTI Turbo Expo held in Atlanta, Georgia, June 2003.

Dey, D. and Camci, C., 2001, "Aerodynamic Tip Desensitization of an Axial Turbine Rotor Using Tip Platform Extensions," ASME paper 2001-GT-484.

Han, S., Han, B., Jin, P. and Goldstein, R.J., 2001, "Numerical Prediction of the Flow Field near the Tip of a Rotating Turbine Blade," Journal of Engineering Physics and Thermophysics, Vol.74, No.4, pp.859-868.

Lakshminarayana, B., Camci, C., Halliwell, I., and Zaccaria, M., 1992, "Investigation of Three Dimensional Flow Field in a Turbine Including Rotor/Stator Interaction. Part I: Design Development and Performance of the Research Facility," AIAA paper 92-3326, presented at the ASME-AIAA Joint Propulsion Conference, Nashville, Tennessee.

Launder,B.E. and Spalding,D.B, 1974, "The Numerical Computation of Turbulent Flows," Comp.Meth.Appl.Mech. Eng. Vol.3,pp-269-289.

Lin, Y.L., Shih, T.I.P., Chyu, M.K. and Bunker, R.S., 2000, "Effects of Gap Leakage on Fluid Flow in a Contoured Turbine Nozzle Guide Vane," ASME paper 2000-GT-0555.

Liu, J. and Bozzola, R., 1993, "Three-Dimensional NavierStokes Analysis of Tip Clearance Flow in Linear Turbine Cascades," AIAA Journal, Vol.31, pp.2068-2074.

Morphis, G. and Bindon, J.P., 1988, "The Effects of Relative Motion, Blade Edge Radius and Gap Size on the Blade Tip Distribution in an Annular Turbine Cascade with Tip Clearance," ASME paper 88-GT-256.

Sell, M., Treiber, M., Casciaro, C. and Gyarmathy, G., 2000, "Tip-clearance-affected Flow Fields in a Turbine Blade Row," Proc. Inst. Mech. Eng., Vol.213, Part A, pp.309, 318.

Tallman, J. and Lakshminarayana, B., 2001, " Numerical Simulation of Tip Leakage Flows in Axial Flow Turbines, with Emphasis on Flow Physics: Part I - Effect of Tip Clearance Height," ASME Journal of Turbomachinery, Vol.123, pp.314-323.

Tallman, J. and Lakshminarayana, B., 2001, " Numerical Simulation of Tip Leakage Flows in Axial Flow Turbines, with Emphasis on Flow Physics: Part II - Effect of Outer Casing Relative Motion," ASME Journal of Turbomachinery, Vol.123, pp.324-333. 